\title{
Dental Caries Diagnosis in X-ray Images using KNN Classifier
}

\author{
V. Geetha ${ }^{1 *}$ and K. S. Aprameya ${ }^{2}$ \\ 'Department of Electronics and Communication Engineering, \\ U.B.D.T. College of Engineering, Davanagere - 577004, Karnataka, India; \\ gvespdvg@gmail.com \\ ${ }^{2}$ Department of Electrical and Electronics Engineering, \\ U.B.D.T. College of Engineering, Davanagere - 577004, \\ Karnataka,India; aprameya_ks@yahoo.com
}

\begin{abstract}
Objectives: Early diagnosis of dental caries helps in maintaining good oral health. The current study focuses on diagnosis of dental caries in dental radiographs through machine learning. Methods: Dental radiographic images in bmp format are considered for study. The images are trained, validated and tested with 10 -fold cross validation. Diagnosis method involves Laplacian filter, adaptive thresholding, morphological transformation, Grey Level Co-occurrence Matrix (GLCM) based texture analysis and K Nearest Neighbors (KNN) Classifier. The diagnostic performance measures accuracy, False Positive Ratio (FPR), precision, recall, Mathews Correlation Coefficient (MCC), Receiver Operating Characteristic (ROC) area are calculated for detection and diagnosis of dental caries. Findings: Proposed method is giving better performance of $98.5 \%$ accuracy, 98.5\% precision, 4.7\% False Positive Rate (FPR) and 0.953 Receiver Operating Characteristic (ROC) curve area with 10 -fold cross validation. The validity of the results tested using two-way ANOVA, at significant level of $5 \%$, shows that the interaction of proposed method on performance parameter measures is significant. Applications/Improvement: The study highlighted the potential utility of machine learning for detection of dental caries in automated computer assisted diagnosis system. The proposed method provided good performance in detecting caries in dental radiographs. The results suggest that the proposed framework is a promising approach for the automatic detection of dental caries in dental radiographs. The performance of the system can be further improved by high quality and quantity dataset.
\end{abstract}

Keywords: Computer Assisted Diagnosis, Dental Caries, Dental Radiography, Machine Learning

\section{Introduction}

Dental caries has become the most common disease, causes bacterial damage to teeth. Caries which are located in between teeth may not be visible to human eye, at an early stage. With the help of dental X-ray image analysis, it is possible to detect dental caries at an early stage. Quantum, photons, electronic and quantization noises degrade dental $\mathrm{X}$-ray images ${ }^{1}$. The 'artefact' on X-ray image appear as light or dark spots, lines, fogging, specks etc. are caused by motion, poor contrast. To improve both contrast and intensity simultaneously, computer aided image processing algorithms can be used ${ }^{2}$. Dental X-ray images consist of teeth areas with highest intensity, bone areas with average intensity and background with lowest intensity. In case of uneven exposure, it is difficult to distinguish between tooth and bone areas $\underline{3}$. Hence pre-processing of dental radiographs are essential to sharpen the boundaries of dental caries and to increase the contrast between image background and tooth. Segmentation of teeth is a significant problem due to teeth variation in shape, size and arrangement of teeth varies between one person to another ${ }^{4}$.

*Author for correspondence 
Gaussian filter and morphological top-hat and bottom hat operations to reduce noise and smoothen the image. The enhanced image is segmented using otsu thresholding, watershed operator and morphological close operation $s^{5}$. Used top-hat and bottom-hat morphological operators to enhance original images, adaptive thresholding to segment the dental X-ray images ${ }^{6,7}$. Iterative thresholding followed by adaptive thresholding ${ }^{8}$, variation level set $^{9}$ segmentation can also be used for segmentation of teeth. Approach for computer aided dental X-ray analysis. Enhancement method that combines homomorphic filtering, adaptive contrast stretching based homogeneity and adaptive morphological transformations can be used for enhancement of dental radiographs ${ }^{10}$. GLCM features extracted from dental radiographs can be used to classify dental cysts using $\mathrm{K}$-means classifier ${ }^{11}$. Automating the process of analysis of dental radiograph is necessary to improve dental procedures. In this study, dental caries diagnosis system based on textural features and KNN classifier used to classify caries or normal dental images. The proposed methodology, experimental results, discussions and conclusion are presented in the following sections.

\section{Methodology}

In this study, diagnosis of dental caries is presented using Laplacian filter, adaptive thresholding, GLCM based texture analysis and KNearest Neighbors (KNN) Classifier. The system is implemented using MATLAB 2017a. The system is validated using 10 -fold cross validation using WEKA (Waikato Environment for Knowledge Analysis).

\subsection{Experimental Dataset}

Dataset for training and testing comprises of 49 caries and 16 normal dental X-ray images. The images are taken from SJM Dental College, India using Gendex X-ray machine with RVG sensor of the type Sirona. The caries in the dental images are annotated by a dentist.

\subsection{Pre-processing}

The dental X-ray images are converted to bmp format using MATLAB conversion tool application. After the conversion, resized to $256 \times 256$ of class double. The resultant image is enhanced using Laplacian filter. Convolving the Laplacian mask with the image, highlights the edges of the image and removes the low frequency components such as contrast present in the image.

\subsection{Segmentation}

Image segmentation is partitioning of an image into multiple sub-regions based on the desired feature. Here, adaptive thresholding is used for segmentation. The assumption behind adaptive thresholding is that smaller regions in an image are more likely to have approximately uniform illumination, suitable for thresholding. The median value of each sub image is taken as optimum threshold. The resultant image is dilated and eroded, and then eroded image is subtracted from the dilated image to get the segmented image.

\subsection{Feature Extractions}

The segmented image is resized to $100 \times 100$ and fed to feature extraction stage. 22 textural features of the segmented image are extracted using GLCM technique and stored in the database. The extracted features include contrast, correlation, energy, homogeneity, mean and entropy.

\subsection{KNN Classifier}

K Nearest Neighbors Classifier is a simple, efficient and nonparametric classifier used for pattern recognition. The classification of samples is done based on the class of their nearest neighbour. KNN is used to classify normal or caries images.

\subsection{K-fold Cross Validation}

Overfitting problem can be avoided by incorporating K-fold cross validation. Then classifier accuracy becomes independent of dataset. In the proposed method performance measures are evaluated by using 10 cross validation technique using WEKA tool (version 3.8.2). The Figure 1 shows the proposed architecture for identification of dental caries. The system consists of two stages: Training and testing stage. Digital dental X-rays images of the training set are passed through Laplacian filter and then segmented using adaptive thresholding. 22 Textural features of the training images are extracted using GLCM. Then best features are selected and stored in the database. To detect the presence of the caries in the test image, the test image is enhanced using Laplacian filter and adaptive thresholded, and textural features are extracted, and only best features are selected and applied to KNN classifier. By using database, the classifier identifies the given test image as caries or normal image. 


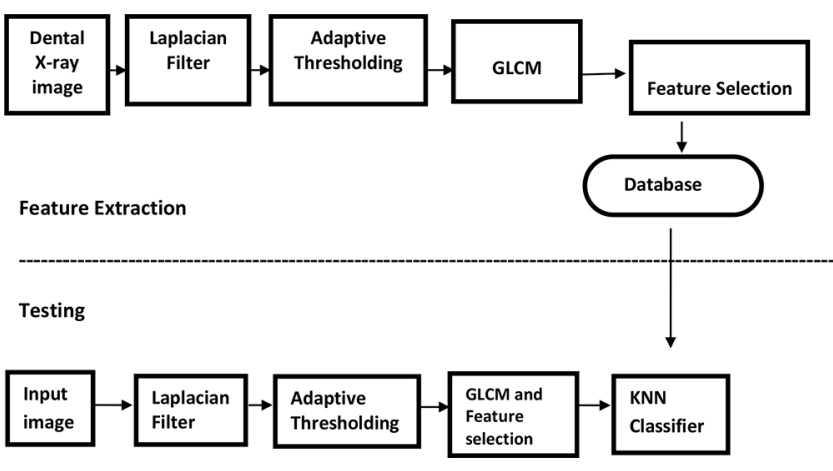

Figure 1. Block diagram of proposed architecture.

\section{Results and Discussion}

The original image, enhanced image using Laplacian filter are shown in Figure 2(a) and Figure 2(b) respectively. The enhanced image has edges only around tooth and caries region and remaining portion of the image is blurred. This greatly helps in extracting the features of tooth region. The Figure 3(a) shows the adaptive thresholded image, is eroded and dilated by using morphological processing, which are shown in Figure 3(b) and Figure 3(c) respectively. Figure $3(\mathrm{~d})$ shows segmented image, which is obtained by subtracting eroded image from dilated image. 22 textural features of the segmented images are extracted using GLCM. Extracted textural features of 65 Dental $\mathrm{X}$-ray bmp images are stored in the database. Using KNN classifier, 65 images are tested for caries using 10-fold cross validation. Table 1 gives the comparison of performance measures of proposed method with the different types of classifier, shows that proposed method is giving higher value of accuracy (98.5\%), Precision (98.5\%), Mathews Correlation Coefficient (MCC) (0.959) and lower value of FPR. But ROC Area (0.953), Precision Recall Curve area (PRC Area) (0.966) is slightly lower than Random Forest classifier. Figure 4 shows the comparison of performance measures of proposed method with the different types of classifier. Receiver Operating Characteristic (ROC) curve for the proposed system is shown in Figure 5, which has the area under the ROC curve of 0.953 which shows that the performance of the proposed system is significant.

\subsection{Statistical Analysis}

Two-way ANOVA statistical analysis result shown in Table 2, for comparison of performance of proposed system with other types of classifier at significant level of
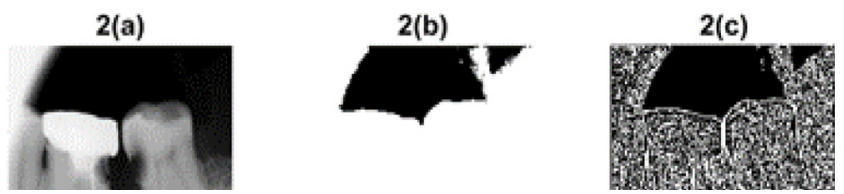

Figure 2. Effect of enhancement on dental image (a) original image in jpg format, (b) original image in bmp format and (c) enhanced image using Laplacian filter.
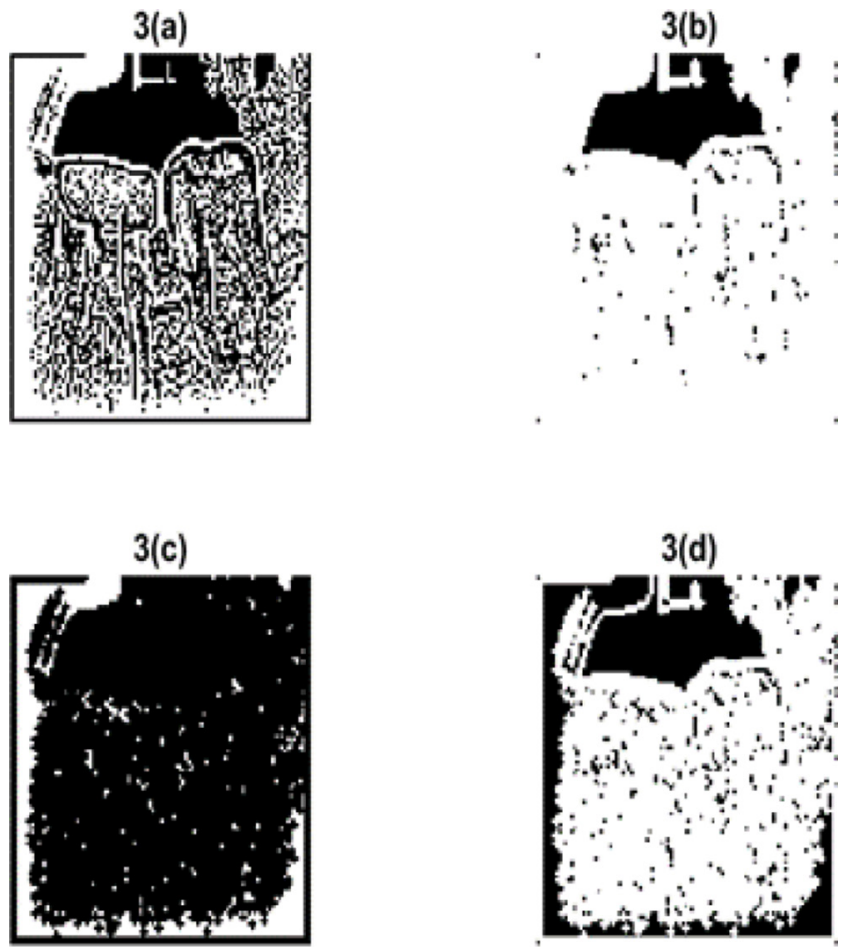

Figure 3. Segmentation results for caries identification (a) adaptive thresholded image, (b) dilated image, (c) eroded image and (d) resultant image after subtracting eroded image from dilated image.

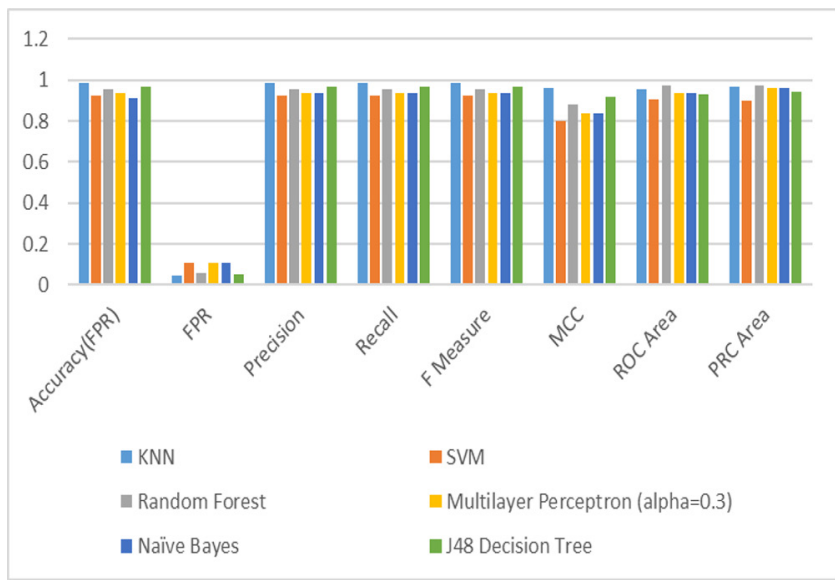

Figure 4. Comparison of performance measures of proposed method with the different types of classifier. 


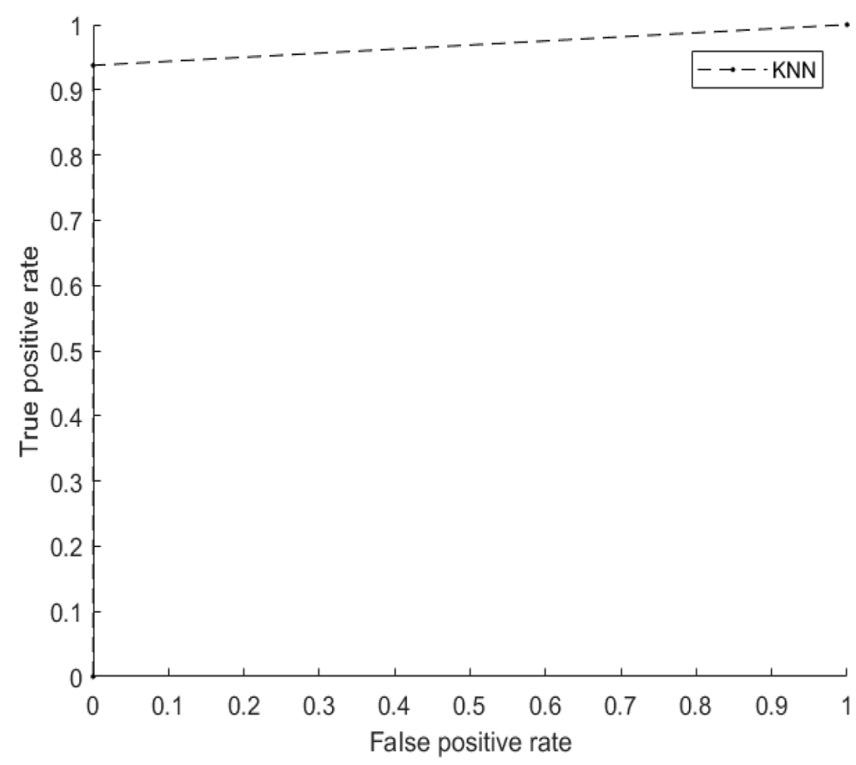

Figure 5. ROC curve for proposed system for Dental caries diagnosis.

$5 \%$, confirms that the interaction of the proposed method on performance parameter measures is significant.

\subsection{Comparison with Other Published Works}

The comparison of proposed work with other previous works is summarized in Table 3. In one of the previous works, features extracted using Principal Component Analysis (PCA) technique and applied to Random Forest classifier and obtained accuracy of $86 \%{ }^{\frac{12}{2}}$. Dental X-ray based tooth caries detection system presented using Histogram of Oriented Gradient (HOG) features and Back Propagation Neural Network Algorithm (BPNN) and achieved an accuracy of $64.91 \% \frac{13}{}$. Clinical X-ray image-based tooth decay diagnosis system using SVM was developed and obtained $86.15 \%$ accuracy for training set and $77.34 \%$ accuracy for test set ${ }^{14}$. In another work, tooth decay diagnosis developed using BPNN achieved an
Table 2. Two-way ANOVA statistical analysis result for the result shown in Table 1 at significant level of 5\%

\begin{tabular}{|c|c|c|c|c|c|}
\hline Source & SS & df & MS & F & Prob $>$ F \\
\hline Columns & 3.899 & 7 & 0.557 & 787.52 & 0 \\
\hline Rows & 0.016 & 5 & 0.003 & 4.41 & 0.0032 \\
\hline Error & 0.025 & 35 & 0.001 & & \\
\hline Total & 3.939 & 47 & & & \\
\hline
\end{tabular}

Table 3. Comparison of caries identification in dental images proposed work with other published methods

\begin{tabular}{|c|c|}
\hline Published work & Accuracy \\
\hline 19 & $0.875 \%$ \\
\hline 18 & $0.935 \%$ \\
\hline 13 & $0.649 \%$ \\
\hline 14 & $0.755 \%$ \\
\hline 15 & $0.942 \%$ \\
\hline 16 & $0.969 \%$ \\
\hline 17 & $0.95 \%$ \\
\hline 12 & $0.86 \%$ \\
\hline Proposed work & $0.985 \%$ \\
\hline
\end{tabular}

accuracy of $94.2 \%$ with 10 hidden layers ${ }^{15}$. Dental caries detection system proposed using digital photographs is capable of detecting caries located on the surface of the tooth enamel and is unable to detect caries which are in between the tooth $\frac{16-18}{}$. A system to classify major dental diseases developed using Convolutional Neural Network $(\mathrm{CNN})^{19}$. Hence the proposed method is concluded as giving higher accuracy than other previously published work.

\section{Conclusion}

In this study, an efficient texture based dental caries diagnostic system is proposed, in which KNN Classifier is used to differentiate caries and normal images. In this

Table 1. Comparison of performance measures of proposed method with the different types of classifier

\begin{tabular}{|l|l|l|l|l|l|l|l|l|}
\hline Methods & accuracy & FPR & Precision & Recall & F Score & MCC & ROC Area & PRC Area \\
\hline KNN & 0.985 & 0.047 & 0.985 & 0.985 & 0.984 & 0.959 & 0.953 & 0.966 \\
\hline SVM & 0.923 & 0.109 & 0.925 & 0.923 & 0.924 & 0.798 & 0.907 & 0.898 \\
\hline Random Forest & 0.954 & 0.057 & 0.955 & 0.954 & 0.954 & 0.879 & 0.973 & 0.974 \\
\hline Multilayer Perceptron (alpha $=0.3)$ & 0.938 & 0.104 & 0.938 & 0.938 & 0.938 & 0.834 & 0.935 & 0.959 \\
\hline Naïve Bayes & 0.908 & 0.104 & 0.938 & 0.938 & 0.938 & 0.834 & 0.935 & 0.959 \\
\hline Decision Tree (J48) & 0.969 & 0.052 & 0.969 & 0.969 & 0.969 & 0.917 & 0.93 & 0.944 \\
\hline
\end{tabular}


system, laplacian filter is used for enhancement, adaptive thresholding to segment the images and features are extracted using GLCM. The experimental results show that caries and normal X-ray images could be distinguished accurately by the diagnostic system. The main advantage of the proposed scheme is its simplicity of implementation, quick computation scheme and easy to operate. Accuracy and reliability of computer assisted dental caries diagnosis system can further improved by using a larger size database. Future work also includes, investigating the method of evaluation of severity of dental caries

\section{Acknowledgements}

Authors would like to thank Dr. R. Gowramma, Principal and Dr. Dharam M Hinduja, Head of the Department, Department of Conservative Dentistry and Endodontics, S.J.M. Dental College, Chitradurga for providing relevant information about dental caries and the datasets used in this research.

\section{References}

1. Gravel P, Beaudoin G, De Guise JA. A method for modelling noise in medical images. IEEE Transactions on Medical Imaging. 2004; 23(10):1221-32.

2. Fundamentals of Medical Imaging. 2009. https://www. amazon.com/Fundamentals-Medical-Imaging-Paul-Suetens/ $\mathrm{dp} / 0521519152$

3. Jain AK, Chen H. Matching of dentalX-ray images for human identification. Pattern Recognition. 2004; 37(7):1519-32. https://doi.org/10.1016/j.patcog.2003.12.016

4. Kondo T, Ong SH, Foong KWC. Tooth segmentation of dental study models using range images. IEEE Transactions on Medical Imaging. 2004; 23(3):350-62. PMid: 15027528. https://doi.org/10.1109/TMI.2004.824235

5. Using the Mathematical Morphology and Shape Matching for Automatic Data Extraction in Dental X-Ray Images. 2013. http://iris.sel.eesc.usp.br/wvc/Anais_WVC2013/Oral/3/1.pdf

6. Zhou J, Abdel M. A content-based system for human identification based on bitewing dental X-ray images. Pattern Recognition. 2005; 3(11):2132-42. https://doi. org/10.1016/j.patcog.2005.01.011

7. Lin PL, Huang PW, Cho YS, Kuo CH. An automatic and effective tooth isolation method for dental radiographs. Opto-Electronics Review. 2013; 21(1):126-36. https://doi. org/10.2478/s11772-012-0051-9

8. Nomir O, AbdelM. A system for human identification for human identification from X-ray dental radiographs. Pattern Recognition. 2005; 38(8):1295-305. https://doi. org/10.1016/j.patcog.2004.12.010
9. Li S, Fevens T, Krzyzak A. An automatic variational level set segmentation framework for computer aided dental X-ray analysis in clinical environments. Computerized Medical Imaging. 2006; 30(2):65-74. PMid: 16500077. https://doi. org/10.1016/j.compmedimag.2005.10.007

10. Lin PL, Lai YH, Huang PW. An effective classification and numbering system for dental bitewing radiographs using teeth region and contour information. Pattern Recognition. 2010; 43(4):1380-92. https://doi.org/10.1016/j.patcog.2009. 10.005

11. Bhan A, Vyas G, Mishra S, Pandey P. Detection and grading severity of caries in dental X-ray images. 2016 International Conference on Micro-Electronics and Telecommunication Engineering; 2016. p. 375-78. https://doi.org/10.1109/ ICMETE.2016.128

12. Singh P, Sehgal P. Automated caries detection based on Radon transformation and DCT. 8th International Conference on Computing, Communication and Networking Technologies; 2017. p. 1-6. https://doi.org/10. 1109/ICCCNT.2017.8204030

13. Albahbah AA, El-Bakry HM, Abd-Elgahany S. Detection of caries in panoramic dental X-ray images using backpropagation neural Network. International Journal of Electronics Communication and Computer Engineering. 2016; 7(5):250-6.

14. Li W, Kuang W, Li Y, Li YJ, Ye WP. Clinical X-ray image based tooth decay diagnosis using SVM. Proceedings of the Sixth International Conference on Machine Learning and Cybernetics. 2007; 3:1616-9. https://doi.org/10.1109/ ICMLC.2007.4370404

15. Yu Y, Li Y, Li YJ, Wang JM, Lin DH, Ye WP. Tooth decay diagnosis using back propagation neural network. Proceedings of the Fifth International Conference on Machine Learning and Cybernetics. 2006. p. 3956-9. https://doi.org/10.1109/ICMLC.2006.258789

16. Olsen GF, Brilliant SS, Primeaux D, Najarian K. An imageprocessing enabled dental caries detection system. 2009 ICME International Conference on Complex Medical Engineering; 2009. p. 1-8. https://doi.org/10.1109/ICCME. 2009.4906674

17. Dental caries detection using NIR imaging technique. 2016. https://digital-library.theiet.org/content/conferences/ 10.1049/cp.2016.1486

18. Datta S, Chaki N. Detection of dental caries lesion at early stage based on image analysis technique. 2015 IEEE International Conference on Computer Graphics, Vision and Information Security; 2015. p. 89-93. https://doi.org/ 10.1109/CGVIS.2015.7449899

19. Prajapati SA, Nagaraj R, Mitra S. Classification of dental diseases using $\mathrm{CNN}$ and transfer learning. 5th International Symposium on Computational and Business Intelligence; 2017. p. 70-4. https://doi.org/10.1109/ISCBI.2017.8053547 